\title{
Der Vergleich von Altem und Neuem Gesetz im Spiegel ausgewählter scholastischer Kommentierungen von 3 Sent., d. 40
}

\author{
Thomas Marschler (Augsburg)
}

\section{Hinführung}

Der Vergleich zwischen Altem und Neuem Gesetz ${ }^{1}$ gehörte bereits vor der Entfaltung eines umfassenden Gesetzestraktats zu den regelmäßig behandelten Themen scholastischer Theologie. Wichtigster Grund dafür war zweifellos das Interesse, das ihm in vielen Texten der Väter (namentlich bei Augustinus) überall dort entgegengebracht worden war, wo einerseits die Überlegenheit der von Christus gestifteten Heilsordnung gegenüber der alttestamentlichen Ordnung aufgezeigt und andererseits die innere Bezogenheit beider aufeinander und dadurch die bleibende Relevanz des Alten Bundes und seines Gesetzes auch im Christentum begründet werden sollten. Das Instrumentarium für diese Auslegung war schon von Theologen vor Augustinus entwickelt worden. Die geistlich-übertragene Interpretation der alttestamentlichen Gesetzestexte geht bis auf Origenes zurück und hatte vor allem über Hieronymus Einzug auch in die westliche Theologie gehalten. Bei Gregor dem Großen wurde sie erneut unterstrichen ${ }^{2}$. In ihrem Licht erweisen die Gesetzestexte des Alten Bundes ihre bleibende Geltung, für deren Anerkennung aber die Christusbotschaft den unverzichtbaren hermeneutischen Schlüssel liefert. Deren größere Dignität in der objektiv-heilsgeschichtlichen Ordnung bildet sich nach der Überzeugung der christlichen Theologen unmittelbar im Vergleich der alt- und neutestamentlichen Texte ab.

Obwohl, wie etwa Beryl Smalley gezeigt hat, die mittelalterliche Theologie schon im 12. Jahrhundert die Bemühung erkennen läßt, das alttestamentliche Gesetz „,in sich“, also im Kontext der heilsgeschichtlichen Situation, in der und

1 Mit V. Marcolino, Das Alte Testament in der Heilsgeschichte. Untersuchung zum dogmatischen Verständnis des Alten Testaments als heilsgeschichtliche Periode nach Alexander von Hales (Beiträge zur Geschichte der Philosophie und Theologie des Mittelalters, N.F. 2), Münster 1970, 85, ist auf die oft sehr weite Verwendung des Begriffs, Gesetz' bei den scholastischen Theologen hinzuweisen: „Die mittelalterliche Scholastik betreibt ihre Theologie des Alten Bundes unter dem Oberbegriff des Gesetzes; so kann das Alte Testament sogar einfach als ,das Gesetz' schlechthin bezeichnet werden."

2 Cf. B. Smalley, William of Auvergne, John of la Rochelle and St. Thomas Aquinas on the Old Law, in: ead., Studies in Medieval Thought and Learning. From Abelard to Wyclif, London 1981, 121-181, hier: 122sq. 
für die es von Gott verfügt worden war, in seinem durch Litteralexegese erfaßbaren Eigenwert wiederzuentdecken ${ }^{3}$, blieb die Vorgabe der Vätertheologie dort wirksam, wo ein unmittelbarer Vergleich zwischen alt- und neutestamentlicher Gesetzesordnung angezielt wurde. Dieser ist nach diversen Aspekten bereits in der Schule von Laon nachweisbar ${ }^{4}$, und er hat seitdem in der frühscholastischen Literatur einen festen Platz eingenommen. Die Behandlung der Gesetze und Sakramente des Alten Bundes ${ }^{5}$ bot sich dabei als geeigneter Ort auch für den Vergleich mit der analog gestalteten Ordnung des Neuen Bundes an ${ }^{6}$.

Schon in den ,Sentenzen' des Petrus Lombardus († 1160) ist unser Thema präsent. Deren drittes Buch behandelt nach der Christologie die drei göttlichen Tugenden und nimmt die Distinktionen über die Liebe zum Anlaß, anschließend weitere handlungsleitende Prinzipien zu besprechen, die für den Christen Relevanz besitzen: die vier Kardinaltugenden (d. 33), die (ebenfalls unter die Tugenden gezählten) sieben Gaben des Heiligen Geistes (d. 34) und nach einigen weiteren Aspekten der Tugendlehre (d. 35-36) die zehn Gebote als Ausfaltung des doppelten Liebesgebotes mit Blick auf Gott und den Nächsten (d. 37-40). Der Dekalog repräsentiert das Zentrum des alttestamentlichen Gesetzes, und in der Behandlung seiner einzelnen Gebote ist immer wieder das Verhältnis zum Neuen Testament zu thematisieren. In d. 40 kommt der Lombarde im Anschluß an die Erläuterung der beiden letzten Weisungen der zweiten Dekalogtafel explizit auf das Verhältnis von „Gesetz und Evangelium“ zu sprechen ${ }^{7}$ Grund dafür scheint zum einen der Inhalt dieser beiden Vorschriften zu sein, das Verbot des Begehrens einer fremden Frau und fremden Eigentums, denn durch ihren Bezug auf die Herzenshaltung und nicht bloß das äußere Werk weisen sie nach Überzeugung des Magisters schon deutlich in die Ordnung des Neuen Bundes hinein. Der Abschluß der Dekalogerklärung lädt darüber hinaus zu einem generellen Fazit über das Verhältnis von Gesetz und Evangelium ein (c. 2 und 3), das Lombardus aus Zitaten des Augustinus und der Glosse zusammenstellt. Damit ist zugleich ein nahtloser Übergang zum vierten Buch geschaffen, das bei der Behandlung der Sakramente den Vergleich der beiden Ordnungen unter vielen Einzelhinsichten fortsetzen wird.

3 Smalley (ibid. 132) nennt als einen möglichen Grund die Herausforderung, welche durch die prinzipielle Gesetzeskritik der Katharer im Raum stand.

4 Cf. die Übersicht bei U. Horst, Gesetz und Evangelium. Das Alte Testament in der Theologie des Robert von Melun (Veröffentlichungen des Grabmann-Institutes zur Erforschung der Mittelalterlichen Theologie und Philosophie, N. F. 13), Paderborn 1971, 1, Anm. 2.

5 Cf. etwa die Kapitel über das alttestamentliche Gesetz bei Hugo von S. Viktor († 1141), De sacramentis, 1. I, p. 12, ed. J.-P. Migne (Patrologiae cursus completus. Series Latina 176), Paris 1854, 347-363, und in der Summa Sententiarum, tr. 4, ed. J.-P. Migne (Patrologiae cursus completus. Series Latina 176), Paris 1854, 118-126.

6 Beispielhaft sei auf die ausführliche Behandlung des Gesetzesvergleichs bei Robert von Melun († 1167) verwiesen, die Ulrich Horst monographisch untersucht hat: Gesetz und Evangelium (nt. 4).

7 Cf. Petrus Lombardus, Sententiae, 1. III, d. 40, c. 2-3 (Spicilegium Bonaventurianum 5), Grottaferrata $1981,229$. 
Die Kommentatoren haben die Vorgabe des Sentenzenmeisters aufgegriffen und gerne die knappen Passagen über das Verhältnis von Gesetz und Evangelium $^{8}$ im Kontext von 3 Sent., d. 40 zu mehr oder weniger ausführlichen Quästionen ausgeformt. Wir beschränken uns im Folgenden auf einige der prominentesten Beispiele dafür aus der Theologie des 13. und frühen 14. Jahrhunderts. Manche dieser Texte sind ausführlicher gestaltet, wie etwa diejenigen des Alexander von Hales OMin ( $†$ 1245), Bonaventura OMin ( $†$ 1274), Thomas von Aquin OP († 1274), Richard von Mediavilla OMin († 1308) oder (mit Einschränkungen) Johannes Duns Scotus OMin († 1308). Andere Autoren der Epoche, wie Albertus Magnus OP († 1280), Petrus Aureoli OMin († 1322) oder Durandus a S. Porciano $\mathrm{OP}(† 1334)$, äußern sich in d. 40 eher kurz, wieder andere auch gar nicht zu unserem Thema, so etwa Richard Fishacre OP ( $†$ 1248), Wilhelm de la Mare OMin († ca. 1285), Robert Kilwardby OP († 1279) und Walter Chatton OMin $(† 1343)^{9}$. Mit der im Verlauf des 14. Jahrhunderts zunehmenden Eingrenzung der Kommentierungen von Buch drei auf wenige, meist sehr spekulative Einzelpunkte wird unsere Fragestellung zurückgedrängt, verschwindet aber bis zum Ende der Sentenzenkommentierungen auf der Schwelle zur Neuzeit niemals völlig, wie etwa ihre Behandlung bei Gabriel Biel († 1495) oder John Mair († 1550) bezeugt ${ }^{10}$. Nur am Rande berücksichtigen können wir im vorliegenden Beitrag die Erörterungen des Themas, die sich außerhalb von Sentenzenkommentaren in teils großer Ausführlichkeit finden, vor allem in scholastischen Summen (wie in den großen Gesetzestraktaten der ,Summa Halensis‘ oder der thomanischen ,Summa theologiae) oder Quästionenfolgen (wie etwa den, Quaestiones de legibus' des Matthaeus ab Aquasparta OMin [† 1302] $)^{11}$.

8 Zur Terminologie cf. U. Kühn, Via Caritatis. Theologie des Gesetzes bei Thomas von Aquin, Göttingen 1965, 70sq. Zunehmend wird lex nova zum Oberbegriff für ,,alles das, was aus dem Munde Christi an Verfügungen, Geboten und Verheißungen als bestimmend für das Leben der an ihn Glaubenden hervorgegangen ist“ (70). Die Bemerkung Kühns, daß damit „die Scholastik mehr und mehr vom Sprachgebrauch Augustins ab[rücke], bei dem ich außer, Evangelium' nur ,Gesetz der Barmherzigkeit‘, ,Gesetz der Liebe‘ und vor allem „Gesetz des Glaubens‘ gefunden habe“ (71), ist nicht ganz korrekt, da der Ausdruck lex nova auch schon bei Augustinus vorkommt; cf. etwa De gratia et libero arbitrio, 18, 37 (PL 44, 903, 44-49); Contra Iulianum opus imperfectum, VI, 15 (CSEL 85/2, 339, 80).

9 Die Formulierung bei U. Kühn, Via caritatis (nt. 8), 74, wonach der Vergleich von Gesetz und Evangelium in d. 40 ,obligat“ zu den Aufgaben der Kommentatoren gehört habe, ist daher übertrieben.

10 Cf. G. Biel, Collectorium circa quattuor libros Sententiarum, 1. III, d. ult., quaest. ult., a. 1-3, edd. W. Werbeck/U. Hofmann, Tübingen 1979, 698-704 (die Ausführungen haben stark kompilatorischen Charakter, sofern sie aus Bonaventura, Thomas oder Scotus genommen sind); J. Maior, In tertium Sententiarum, Paris 1517, hier: d. 40, q. 4 (164ra-va).

11 Natürlich gibt es noch zahlreiche weitere Kontexte, innerhalb derer mittelalterliche Theologen das Thema in teils origineller Gestalt aufgreifen und denen hier ebenfalls nicht weiter nachgegangen werden kann. Beispielhaft genannt seien etwa die stark den Gegensatz von Zeitlichkeit und Ewigkeit reflektierende Auslegung im Johanneskommentar Meister Eckharts (cf. die Belege bei U. Kern, Der Gang der Vernunft bei Meister Eckhart [Rostocker theologische Studien 25], Berlin-Münster 2012, 214sqq.) oder die politisch-theologische Verwertung unseres Gegensatz- 
II. Der Vergleich zwischen Gesetz und Evangelium

\section{Elemente des Vergleichs}

Die üblichen drei Teilaspekte, nach denen die Ordnungen des Alten und Neuen Bundes untersucht und verglichen werden, übernimmt Lombardus aus der Glossentradition $^{12}$ : Sie sind geprägt durch „unterschiedliche Verheißungen“, „unterschiedliche Sakramente“ und „unterschiedliche Gebote“. Auch die allermeisten nachfolgenden Autoren knüpfen an diese Trias an. Innerhalb ihrer gestehen die Scholastiker nur den Moralvorschriften kontinuierliche Fortgeltung im Neuen Bund zu, während die Wirksamkeit der alttestamentlichen Rechts- und Zeremonialvorschriften ihrem Wortlaut nach mit Christi Tod endet. Dennoch bleiben sie nach Lombardus im Evangelium „,in vollerer Weise“ enthalten ${ }^{13}$. Dahinter steht die aus der Vätertheologie bekannte Leitthese, wonach das Evangelium das ganze Gesetz enthält, nicht aber umgekehrt ${ }^{14}$. Diejenigen seiner Weisungen, die im Neuen Bund nicht mehr in wörtlicher Form vorgeschrieben sind, dürfen dennoch als in geistlicher Hinsicht erfüllt und somit in „aufgehobener“ Gestalt präsent angesehen werden.

Dieses Unterscheidungs- und Vergleichsmuster wird auch von den späteren Autoren durchgängig übernommen ${ }^{15}$. Zunehmend vermehrt werden im 13. Jahrhundert die Formalaspekte der Gegenüberstellung. Alexander von Hales präsentiert in seiner Sentenzenglosse ${ }^{16}$ ein nach der aristotelischen Ursachenlehre differenziertes Schema. So unterscheiden sich nach ihm die beiden Ordnungen in der Benennung („Gesetz“ und „Testament"), nach der Wirkursache (Moses und Christus), der Materialursache („fleischliche“ Gebote und Verheißungen gegenüber ,geistlichen“), den angestrebten Zielen und Wirkungen („Befreiung von der Schuld“ gegenüber „umfassender Erlösung“, unvollkommener gegenüber vollkommenem Gehorsam) und der Formalursache („Furcht“ gegenüber „Liebe“).

paares bei Wilhelm von Ockham (De imperatorum et pontificum potestate, in: Guillelmi de Ockham Opera Politica IV, ed. H. S. Offler, Oxford 1997, 261-355, hier: c. 1-3, 282-287; Kernaussage: Die Annahme einer Gewaltenfülle des Papstes in spiritualibus et temporalibus würde dazu führen, daß das Neue Gesetz zur ärgeren Knechtschaft würde als das Alte).

12 Die Herausgeber verweisen auf die Glossa ordinaria zu Hebr 8,6-7, auf Bezüge zur Glossa media zu Ps 73,1 sowie zu Hugo von S. Viktor, De sacramentis, 1. I, p. 12, c. 10 (PL 176), 364A. Cf. auch M.-D. Chenu, La théologie de la loi ancienne selon saint Thomas, in: Revue Thomiste 61 (1961), 485-497, hier: 491sqq.

13 Petrus Lombardus, Sententiae, 1. III, d. 40, c. 2 (Spicilegium Bonaventurianum 5), 229.

14 Alexander von Hales, Glossa in quattuor libros Sententiarum Petri Lombardi, l. III, d. 40 (Bibliotheca Franciscana Scholastica Medii Aevi 14), Quaracchi 1954, 545sq.

15 Bonaventura, Liber III Sententiarum, d. 40, q. 2 c., ed. L. M. Bello (Opera theologica selecta, ed. minor), Quaracchi 1941, 900a-b, verweist auf die Einführung der drei Begriffe bei Hugo von S. Viktor (De sacramentis, 1. I, p. 12, c. 4 [PL 176], 351): ,sacramenta sunt adiutoria, ut per praecepta veniatur ad promissa“.

16 Cf. Alexander von Hales, Glossa, 1. III, d. 40, Quaracchi 1954, 544sq. 
Die ,Summa Halensis` kennt leicht abweichende Vergleichspunkte ${ }^{17}$. In einer stärker konzentrierten, aber im Prinzip unveränderten Form diskutiert Bonaventura unser Thema in drei Quästionen, welche Gesetz und Evangelium in sich, hinsichtlich ihrer Wirkungen sowie der jeweils mit ihnen ,verbundenen Last“ bewerten ${ }^{18}$.

Schon dieser knappe Überblick zeigt: Die Abhandlungen der scholastischen Autoren sind durchweg geprägt von der Konstruktion schroffer Gegensätze, mit deren Hilfe die Differenz zwischen der alten und der neuen Heilsordnung auf möglichst vielen Ebenen hervorgehoben werden soll und die allesamt den Beweis der Überlegenheit des Evangeliums gegenüber dem Alten Gesetz anzielen.

\section{Die Gründe für die Überlegenheit des Evangeliums gegenüber dem Alten Gesetz.}

Wenn die mittelalterlichen Theologen nach den Gründen fragen, die das Gesetz gegenüber dem Evangelium als defizient erscheinen lassen, folgt die Verhältnisbestimmung fast durchgängig dem hermeneutischen Grundprinzip von Verheißung und Erfüllung. Dieses läßt sich in zwei Aussagen exakter entfalten.

(1) Was das Gesetz des Alten Bundes anzielte, vermochte es selbst nicht zu erreichen. Die Gebote, die es vorschrieb, konnten de facto nicht erfüllt werden, sondern führten den Menschen im Gegenteil nur noch tiefer in die Schuld vor Gott. Die negativen Charakterisierungen des Gesetzes als „tötender Buchstabe“ oder „Gesetz der Furcht“, die das Mittelalter vor allem von Augustinus übernommen hat ${ }^{19}$, werden mit Bezug auf diese verderblichen Konsequenzen legitimiert.

(a) Entscheidender Grund für das Scheitern des Gesetzes in seinem wörtlichen Verständnis, so sind alle Scholastiker überzeugt, war das Fehlen oder doch zumindest das unzureichende Maß der Gnade, das mit seiner Befolgung verbunden war. Erst infolge des Sühnetodes Jesu hat Gott diese Erlösungsgnade gewährt. Die Rechtfertigung des Sünders, die im Neuen Bund als eingegossenes Geschenk in der Kraft des Glaubens und durch die Vermittlung der Sakramente gegeben ist, kommt darum nach Meinung der meisten vorher in der Regel nicht zustande ${ }^{20}$. Diese Ansicht ist seit Lombardus in der Schule in vielen Variationen vorgetragen worden $^{21}$. Thomas von Aquin unterstreicht sie besonders deutlich, wenn er in seiner ,Summa“ das „Neue Gesetz“ des Evangeliums geradezu mit der rechtfertigen-

17 Cf. Summa fratris Alexandri [Summa Halensis], tom. IV, Quaracchi 1948, 1. III, n. 547 (846a-b); n. 549 (848a-850b).

18 Cf. Bonaventura, 3 Sent., d. 40, q. 1-3, ed. Bello (nt. 15), 896-904.

19 Cf. etwa A. Guindon, La pédagogie de la crainte dans l'histoire du salut selon Thomas d'Aquin, Paris-Tournai 1975, 25-28.

20 Es fehlte die solutio pretii: Thomas von Aquin, Scriptum super Sententiis magistri Petri Lombardi, d. 40, a. 4, qa. 1, sol., ed. M. F. Moos, vol. 3, Paris 1933, 1310; cf. auch Summa theologiae, I-II, q. 107, a. 2 c., Rom 1962, 1042.

21 Cf. A.M. Landgraf, Die Gnadenökonomie des Alten Bundes, in: id., Dogmengeschichte der Frühscholastik, vol. III/1, Regensburg 1954, 19-60, hier bes. 39-52, der auch auf differenzierende Thesen in der frühscholastischen Epoche hinweist. 
den „Gnade des Heiligen Geistes, die im Glauben an Christus verliehen wird“, identifiziert $^{22}$. Mit diesen Ausführungen über die lex nova als lex gratiae überschreitet Thomas nicht nur terminologisch die Vorgaben seiner Zeitgenossen ${ }^{23}$, sondern erweist sich auch in der Charakterisierung des mit dem Begriff Bezeichneten als innovativ. Es geht um ein Gesetz als lebendiges, von Gott geschenktes Handlungsprinzip, das nun aus dem Inneren des Menschen heraus seine Wirksamkeit entfaltet ${ }^{24}$; Weisung und Hilfe fallen in ihm zusammen. Schon in seinem Sentenzenkommentar charakterisiert Thomas demgegenüber das geschriebene Gesetz des Alten Bundes als bloßes Zeichen der angestrebten, im Neuen Bund realisierten Gerechtigkeit vor Gott, dem echte Wirksamkeit fehlte und das bestenfalls dispositive Kraft zu entfalten vermochte ${ }^{25}$. Seine Problematik lag in „der Diskrepanz zwischen Lehre und Kraft" ${ }^{\text {"26 }}$. Andere Autoren haben sich zur rechtfertigenden Kraft des Alten Gesetzes im Kontext des unmittelbaren Vergleichs etwas positiver geäußert, ohne diese Position grundsätzlich zu verändern. Durandus etwa spricht nicht bloß von der vorbereitenden Funktion, welche das Alte Gesetz in seiner Geltungszeit durch Rechts- und Kultvorschriften ausübte, sondern möchte ihm sogar eigentlich rechtfertigende Kraft hinsichtlich der eingegossenen Gnade zugestehen, wenn auch nicht in wirkursächlicher, sondern allein ,,in verdienstursächlicher Form“, und zwar de congruo, nicht de condigno (also in einer Weise des Verdienstes, die nicht in vollkommener Entsprechung zum Verdienten, der Rechtfertigungsgnade, steht, wohl aber in einem vor Gott relevanten unvollkommenen Entsprechungsverhältnis ${ }^{27}$. In solchen Thesen setzt sich die schon in der Frühscholastik entwickelte Meinung fort, daß im Alten Bund ,nicht das opus

22 Thomas, Summa theologiae, I-II, q. 106, a. 1, c., Rom 1962, 1037: „Id autem quod potissimum est in lege Novi Testamenti, et in quo tota virtus eius consistit, est gratia Spiritus Sancti, quae datur per fidem Christi". Cf. zur Charakterisierung der thomanischen lex nova den Kommentar von Th. Deman, in: Deutsche Thomas-Ausgabe, vol. 14: Der neue Bund und die Gnade, Heidelberg e. a. 1955, 287-325. Die Originalität und Bedeutung dieser Konzeption, nicht zuletzt in ökumenischer Perspektive, hat Kühn, Via Caritatis (nt. 8), bes. 163-223, herausgearbeitet. Zum Verhältnis von „Altem und Neuem Gesetz" im thomanischen Sentenzenkommentar cf. ibid. 70-79. Siehe auch B. Ippolito, Iustificatio e Lex Nova nella Summa Theologiae, in: F. Di Blasi (ed.), Riscoprire le radici e i valori comuni della civiltà occidentale. Il concetto di legge in Tommaso d'Aquino, Palermo 2007, 157-174.

23 Cf. Marcolino, Das Alte Testament (nt. 1), 295.

24 Cf. dazu P. Rodriguez, Spontanéité et caractère légal de la Loi nouvelle, in: L. J. Elders/K. Hedwig (eds.), Lex et libertas. Freedom and Law according to St. Thomas Aquinas. Proceedings of the fourth Symposium on St. Thomas Aquinas' Philosophy, Rolduc, Nov. 8 and 9, 1986, Città del Vaticano 1987, 254-264.

25 Cf. Thomas, 3 Sent., d. 40, a. 3 c., ed. Moos (nt. 20), 1306 sq., und ad 2, 1307.

26 Kühn, Via Caritatis (nt. 8), 72. Letztlich legen die Scholastiker durchgängig das Wort Augustins, e. a. vorgetragen in De spiritu et littera (c. 13, n. 22, ed. K.F. Urba [Corpus Scriptorum Ecclesiasticorum Latinorum 60], Wien 1913 [Nachdruck Wien 1962], 175), aus, das die Summa Halensis (1. III, n. 554, Quaracchi 1948, 858b) auch wörtlich anführt: „Per legem operum dicit Deus homini: Fac quod iubeo; per legem fidei dicit homo Deo: Da quod iubes."

27 Cf. Durandus, Super tertium Sententiarum, Paris 1550, d. 40, q. 3, 247vb. 
operatum, wohl aber das opus operantis rechtfertigte bzw. verdienstlich war“ ${ }^{28}$, näherhin unter der Voraussetzung, daß das Gesetz seinem geistlichen Sinn nach erkannt und befolgt wurde. Ähnlich äußert sich Richard von Mediavilla, der allerdings als Bedingung nennt, daß neben der geistlichen Beobachtung des Gesetzes ein expliziter oder impliziter Christusglaube vorgelegen haben muß, damit das Verdienst einer iustitia infusa eingeräumt werden $\mathrm{kann}^{29}$. Solche Aussagen zur rechtfertigenden Kraft des Gesetzes unter ganz bestimmten Bedingungen sind auch bei anderen Scholastikern zu finden, bei Thomas von Aquin vor allem in seinen Schriftkommentaren ${ }^{30}$. Unter diesen Vorgaben mußte man die Heilswirkung des Alten Gesetzes allerdings faktisch auf einen äußerst kleinen Kreis von Menschen beschränkt sehen ${ }^{31}$.

(b) Im Regelfall hat nach Meinung der Scholastiker das Gesetz den Menschen vor Gott nicht gerecht gemacht, sondern tatsächlich das Gegenteil bewirkt: „,Es vermehrt“, wie bereits Lombardus mit Verweis auf Augustinus schreibt, „die Konkupis-

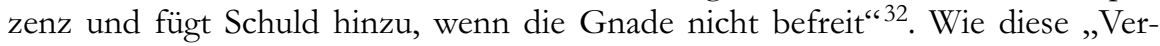
mehrung von Schuld“ zu verstehen ist, wird von den Autoren nach mehreren Aspekten beschrieben, die Alexander von Hales in eine geordnete Aufzählung faßt:

„Es heißt, das Gesetz des Mose decke die Schuld auf, bringe Zorn hervor, vermehre die Sünde und wecke Furcht. Diese Wirkungen aber stehen in einer Ordnung: Das Erste nämlich ist die Erkenntnis der Sünde, als Zweites kommt die Weckung von Furcht, das Dritte ist das Verbot der Sünde, daß sie nicht geschehen soll; viertens aber folgt, daß, wenn sie geschieht, sie vermehrt wird; an fünfter Stelle steht, daß es [das Gesetz] nach vollzogener Handlung Sünde hervorbringt, das bedeutet: Strafe für die Sünde.“33

${ }^{28}$ Landgraf, Die Gnadenökonomie (nt. 21), 60.

${ }^{29}$ Cf. Richard von Mediavilla, Super quatuor libros sententiarum Petri Lombardi, 3 Sent., d. 40, a. 1, q. 4 c., Brixen 1591 [Nachdruck Frankfurt 1963], 482b.

${ }^{30}$ Nachweise bei O. H. Pesch, Kommentar in: Deutsche Thomas-Ausgabe, vol. 13: Das Gesetz, Heidelberg e. a. 1977, 529-734, hier: 674-678 („,Heil unter dem Gesetz“).

31 Die generelle Partikularität des Alten Bundes und Gesetzes für Israel gegenüber der Universalisierung des Heilsangebotes für alle Menschen spielt in den Vergleichen unserer Autoren eine erstaunlich geringe Rolle. Ausdrücklich verwiesen sei in diesem Zusammenhang auf Matthaeus ab Aquasparta, Quaestiones de legibus, in: Quaestiones disputatae de anima separata, de anima beata, de ieiunio et de legibus, ed. C. Piana (Bibliotheca Franciscana Scholastica Medii Aevi 18), Quaracchi 1959, 429-571, hier: q. 5 c., 526.531. Ein Argument, warum das Alte nicht mit dem Neuen Gesetz gemeinsam fortbestehen konnte, lautet: „Secunda ratio est propter universalis legis traditionem. Sicut enim dictum est, illa Lex fuit particularis, uni tantum populo tradita et in illa remedia efficacia tantum uni sexui. De iis autem quae ad salutem pertinent vel sunt necessaria ad salutem, praecepta communia et universalia leges particulares et praecepta evacuant; aliter iniuste ageretur cum eis quibus particularia sunt data praecepta, cum ad plura necessaria quam alii tenerentur; et peior esset conditio Iudaeorum quam gentium, cum ad eorum iustificationem plura necessaria exigerentur; quod constat esse falsum" (531).

32 Petrus Lombardus, Sententiae, 1. III, d. 40, c. 2, 229. Nach Lombardus war die Gnade im Gesetz nicht so reich vorhanden wie im Evangelium (,non sic abundabat"); damit wird sie immerhin dem Gesetz nicht völlig abgestritten.

33 Alexander von Hales, Glossa, 1. III, d. 40, Quaracchi 1954, 549: „Dicitur autem Lex Moysi ostendere peccatum, operari iram, angere peccatum, inducere timorem. Sunt autem isti effectus ordinati: primum enim est cognitio peccati; secundum est inducere timorem; tertium est probibere peccatum ne fiat; quartum est, si fiat, quod angetur; quintum, si actum est, operatur peccatum, id est poenam peccati." 
Diese Momente tauchen auch bei nachfolgenden Theologen in verschiedener Zusammenstellung und gelegentlich mit leichten Akzentverschiebungen auf. So sehen etwa Albert und Thomas von Aquin den erweiterten Strafcharakter darin mitbegründet, daß aus dem vorher nur naturrechtlich Verbotenen durch das Gesetz auch positiv-rechtlich Untersagtes wird ${ }^{34}$.

(c) Ziel der Autoren ist es stets, die im Ergebnis negative Wirkung des Gesetzes konstatieren zu können, ohne dessen innere Schlechtigkeit behaupten zu müssen, weil dies den göttlichen Ursprung der lex vetus zweifelhaft machen könnte. Für die Explikation dieses Zusammenhangs bot die paulinische Theologie eine entscheidende Vorlage: Es war das Sündersein des Menschen, das Leben im Status des Nicht-Begnadetseins ${ }^{35}$, das die an sich guten Weisungen des Gesetzes in ihr Gegenteil verkehrte. Es „befiehlt, was ohne die Gnade nicht erfüllt werden kann“"36. Das Scheitern des Menschen am Gesetz ist folglich nicht diesem selbst anzukreiden. Zum „tötenden Gesetz“, so Bonaventura oder ähnlich Albertus Magnus, wird es per occasionem, nicht per causam bzw. per se ${ }^{37}$. Es wirkt nach dem Wort der Glosse beiläufig tödlich, wie ein Schwert, das jemand zur eigenen Verteidigung trägt, das aber dann ein Feind benutzt, um den Träger umzubringen ${ }^{38}$. Das Gesetz, so Thomas von Aquin, ist ebensowenig schuld daran, daß die Sünder an ihm scheitern, wie die Sonne, wenn Fiebernde sterben, die ihr ausgesetzt sind ${ }^{39}$. Einen ähnlichen Vergleich kennt auch Albert: Das Gesetz wirkte in seiner die Sünde offenlegenden, sie aber nicht eindämmenden Kraft wie Wasser, das den Durst eines Dürstenden noch vermehrt, wenn es ihm gezeigt wird ${ }^{40}$. Zu beseitigen vermag es ihn nicht.

(2) Aber nicht nur nach der realen Möglichkeit seiner Erfüllung, sondern auch in inhaltlicher Hinsicht gilt das alttestamentliche Gesetz für die von uns untersuchten Theologen als defizient.

34 Cf. Albertus Magnus, Commentarii in III Sententiarum, d. 40, a. 4 c., ed. Borgnet (Opera omnia 28), Paris 1894, 752a-b; Thomas, 3 Sent., d. 40, a. 3 c., ed. Moos (nt. 20), 1306sq.

35 Alexander von Hales verweist ausdrücklich auf die Erbsünde: Alexander von Hales, Glossa, 1. III, d. 40, Quaracchi 1954, 549.

36 Petrus Lombardus, Sententiae, III, d. 40, 229.

37 Cf. Bonaventura, 3 Sent., d. 40, q. 2 c., ed. Bello (nt. 15), 900b; Albert, 3 Sent., d. 40, a. 4 c., ed. Borgnet (nt. 34), 752a. Mit Bezug auf ähnliche Gedanken bei Alexander von Hales kommentiert Marcolino, Das Alte Testament (nt. 1), 111sq.: „Die Aufgabe des Gesetzes war es also, Israel zu veranlassen, die Sünde zu meiden, und es so auf den Wegen Gottes zu führen. Welche Wirkung aber hatte das Gesetz in Wirklichkeit? Wenn es sich auch als heilige Forderung Gottes behauptete, so trug es in seiner geschichtlichen Auswirkung nur zur Vermehrung der Sünde bei; doch gerade darin bestand eine seiner heilsgeschichtlichen Funktionen."

38 Cf. Bonaventura, 3 Sent., d. 40, q. 2 c., ed. Bello (nt. 15), 900b-901a.

39 Cf. Thomas, 3 Sent., d. 40, q. 1, a. 3 ad 4, ed. Moos (nt. 20), 1308. Cf. Pesch, Kommentar (nt. 30), 669sqq. (,Thomas über das ,tötende Gesetz“).

40 Cf. Albert, 3 Sent., d. 40, a. 4 c., ed. Borgnet (nt. 34), 752a-b: ,sicut quando febricitanti aqua ostenditur, magis excitatur in eo sitis quam prius: et ita etiam aestuanti bomini in concupiscentia, decalogus eo ipso quod probibet, ostendit peccatum: et cum non dat gratiam, est occasio provocationis concupiscentiae: et hic est unus modus occidendi.“ 
(a) Zum Vorwurf gegen Gott kann jedoch diese Feststellung nach ihrer Ansicht ebenfalls nicht gewendet werden, denn das Gesetz war immer schon auf seine Überbietung durch das Evangelium angelegt ${ }^{41}$. Es besaß die Funktion eines (Vor-)Zeichens, das auf die noch ausstehende Erfüllung, die größere Hoffnung verweist ${ }^{42}$. „Das Gesetz wurde gegeben, auf daß die Gnade gesucht würde“, hatte schon Augustinus festgestellt ${ }^{43}$, und unter dieser Prämisse konnte man selbst problematisch erscheinende Elemente seines Inhalts sinnvoll interpretieren $^{44}$. Das Schema von Verheißung und Erfüllung ist somit das Grundprinzip des gesamten scholastischen Gesetzesvergleiches und wird von den Autoren gerne in differenzierter Weise herangezogen.

(b) Die Überlegenheit des Evangeliums auf der Ebene der Gebote wird sowohl mit Blick auf ihren im Evangelium stark verminderten Umfang als auch auf Veränderungen in ihrer Qualität begründet. Während der erste Punkt kaum eingehenderer Erläuterung bedurfte, hat man zum zweiten Aspekt diverse Explikationsversuche vorgetragen ${ }^{45}$. So seien die Weisungen des Alten Bundes vorrangig am Maßstab der Gerechtigkeit orientiert gewesen, während im Evangelium ausgleichend die Barmherzigkeit hinzugekommen sei ${ }^{46}$. Für Bonaventura zeigt sich der unterschiedliche Charakter von Gesetz und Evangelium bereits im jeweiligen Modus ihrer Einsetzung: Während die Übergabe des Alten Gesetzes „unter großem Getöse und Furcht“" stattfand, wurde das Evangelium mit „großer Güte und Süßigkeit" verkündet ${ }^{47}$. Diese Differenz betont der Franziskaner auffällig stark, aber ähnliche Hinweise auf die Milde des göttlichen Gesetzgebers im Neuen Bund kennen auch die ,Summa Halensis ${ }^{448}$ und im Rahmen von 3 Sent., d. 40 Thomas $^{49}$ und Aureoli ${ }^{50}$. Die Differenz der Gesetze angesichts ihrer Prägung durch Furcht und Strafandrohung einerseits und Liebe andererseits wird regelmäßig auf die Unterschiedlichkeit der jeweiligen Adressaten zurückgeführt. Während, so Bonaventura, im Alten Bund ,hartnäckige Menschen, die zum Bösen geneigt waren“, durch Strafandrohungen gezähmt werden sollten, sind nach dem

41 Cf. ausdrücklich Alexander von Hales, Glossa, l. III, d. 40, Quaracchi 1954, 546.

42 Cf. etwa Thomas, 3 Sent., d. 40, q. 1, a. 4, ed. Moos (nt. 20), 1308.

3 „Lex data est, ut gratia quaereretur": Augustinus, De spiritu et littera 19,34 (CSEL 60), 186sq.

44 Cf. Marcolino, Das Alte Testament (nt. 1), 115-122.

45 Cf. zu den frühscholastischen Vorgaben Landgraf, Die Gnadenökonomie (nt. 21), 34sqq.

46 Cf. Alexander von Hales, Glossa, l. III, d. 40, Quaracchi 1954, 546.

47 Bonaventura, 3 Sent., d. 40. q. 1 c., ed. Bello (nt. 15), 897b.

48 Cf. Summa Halensis, 1. III, n. 552, Quaracchi 1948, 854a-856b.

49 Cf. Thomas, 3 Sent., d. 40, a. 4, qa. 2, sol., ed. Moos (nt. 20), 1311. Weitere Aspekte benennt M. Qualizza, Israele nella storia della salvezza secondo san Tommaso d'Aquino (Publicazioni del Pontificio Seminario Lombardo in Roma, Ricerche di Scienze Teologiche, Ser. min. 4), Brescia 1978, 90sqq. Zur Unterscheidung von timor servilis und timor filialis, die Thomas hier anwendet, cf. Guindon, La pédagogie de la crainte (nt. 19), 62-104.

50 Cf. Petrus Aureoli, Commentaria in tertium librum Sententiarum, d. 40, q. ult., a. un., Rom 1605, 541b. Er verweist bei der Diskussion von timor und amor auf die Promulgation des Neuen Gesetzes durch den am Kreuz hängenden Christus und auf die Tatsache, daß das neue Gesetz am Pfingsttag in die Herzen der Apostel geschrieben wurde. 
Erscheinen der Menschenfreundlichkeit Christi die Menschen „für Überzeugung offen" geworden (persuadibiles) und können daher durch Verheißungen gelenkt werden, die man zu lieben und zu ersehnen vermag und die zum Tun des Guten Anreiz geben ${ }^{51}$. Das Volk des Alten Bundes wird in diesem Zusammenhang stets als ein unreifes, irdisch denkendes charakterisiert, das aus pädagogischer Sicht einer härteren Hand bedurfte - wie nach den Worten des Aquinaten Kranke strengere Diät halten müssen als Gesunde und Kinder einer härteren Disziplin unterworfen werden als Erwachsene ${ }^{52}$. Der Weg vom Alten zum Neuen Gesetz erscheint somit als langsam entfalteter göttlicher Erziehungsprozeß, in dem Gott das für die jeweils Angesprochenen Beste besorgt und Christus die entscheidende Wende gebracht hat ${ }^{53}$.

(3) Im Kontext dieser Vergleiche meldet sich eine Reihe von Einwänden, die von den Autoren in unterschiedlicher Kombination diskutiert werden.

(a) Häufig beschäftigt man sich mit der Frage, ob die Überlegenheit des Evangeliums nicht dadurch gefährdet wird, daß es in bestimmten Punkten das Alte Gesetz verschärft und Hinzufügungen vorgenommen hat. Bonaventura bewertet die Verteilung der „Last des Gesetzes“ folgendermaßen ${ }^{54}$ : Schwerer zu erfüllen ist das Neue Gesetz nicht der Quantität, wohl aber der Qualität der Forderungen nach, da es anders als das Alte auf die Vollkommenheit des Menschen abzielt ${ }^{55}$. Dagegen steht jedoch eine klare Erleichterung auf zwei anderen Ebenen: hinsichtlich der „,bewegenden Affekte“, weil nun Liebe statt Furcht wirksam ist, und hinsichtlich der „,helfenden Sakramente“, die im Neuen Bund anders als im Alten weitaus mehr Nutzen als Schwierigkeit mit sich bringen. Gnade und Liebe gleichen also alle scheinbaren Mehrbelastungen des Neuen Bundes aus ${ }^{56}$. Was immer

51 Cf. Bonaventura, 3 Sent., d. 40. q. 1 c., ed. Bello (nt. 15), 897b.

52 Cf. Thomas, 3 Sent., d. 40, a. 4, qa. 3 ad 3, ed. Moos (nt. 20), 1312. Thomas hat den pädagogischen Charakter der Furcht im Alten Bund im Verlauf seines Werkes zunehmend deutlicher herausgestellt. Cf. den detaillierten Nachweis bei Guindon, La pédagogie de la crainte (nt. 19); weitere Hinweise bietet auch Qualizza, Israele nella storia della salvezza (nt. 49).

53 Schon zu Beginn des 13. Jahrhunderts hat, wie Smalley, William of Auvergne (nt. 2), 137-156, zeigt, die Betonung des pädagogischen Charakters der alttestamentlichen Weisungen bei Wilhelm von Auvergne das zweite aus der Väterzeit übernommene Deutungsmotiv, das die lex vetus als schattenhaftes Vorzeichen der Gnadenordnung des Neuen Bundes ansieht, ganz in den Hintergrund drängen können. Auf eine allegorische Auslegung der entsprechenden alttestamentlichen Texte konnte dann völlig verzichtet werden. Dieses Vorgehen setzte sich aber, wie auch die von uns behandelten späteren Autoren des 13. Jahrhunderts belegen, nicht durch.

54 Cf. Bonaventura, 3 Sent., d. 40, q. 3, ed. Bello (nt. 15), 903 a.

55 Cf. ähnlich schon Wilhelm von Auxerre, Summa aurea, 1. IV, tr. 2, c. 2 sol., ed. J. Ribaillier (Spicilegium Bonaventurianum 20), Paris-Rom 1987, 22, Z. 55-58: „Duplex est difficultas sive gravitas: una carnalis, alia spiritualis. Quantum ad gravitatem carnalem gravior erat lex vetus quam evangelium; sed quantum ad difficultatem spiritualem lex nova difficilior est." Die Bezeichnung des alttestamentlichen Gesetzes als lex vetus übernimmt auch Alexander von Hales (Marcolino, Das Alte Testament [nt. 1], 87).

56 Bereits Alexander von Hales verweist auf das Wort des Augustinus, wonach jede Bürde für den Liebenden leicht zu tragen sei (Glossa, l. III, d. 40, Quaracchi 1954, 547); cf. auch Thomas, Summa theologiae, I-II, q. 107, a. 4 c., Rom 1962, 1044; Johannes Duns Scotus, Ordinatio. Liber 
hier hinzugefügt worden sein mag, so ist sich Bonaventura sicher, dient nur dem Fortschritt in der Liebe und ist darum letztlich als Erleichterung zu bewerten, mag es auch auf den ersten Blick als Erschwernis erscheinen - „wie die Federn beim Vogel oder das Segel beim Schiff“"57. Thomas von Aquin geht auf diese Hinzufügungen inhaltlich näher ein. Als Beispiele nennt er die mit dem Vollkommenheitsideal verbundenen evangelischen Räte, denen allerdings keine strenge Verpflichtung zukommt, und bestimmte Verschärfungen, die mit der exakteren Erklärung der Sittengebote, wie sie Jesus vorgenommen hat, im Zusammenhang stehen, darunter etwa die Rücknahme der Scheidungserlaubnis ${ }^{58}$. Damit greift er exakt diejenigen Punkte auf (consilia und explanatio [legis]), die am Beginn der Hochscholastik bereits Wilhelm von Auxerre als neutestamentliche Erweiterungen des Gesetzes auf geistlicher Ebene vermerkt hatte ${ }^{59}$. Für Richard von Mediavilla hat Christus in diesen Fällen bloß äußere Zeremonien außer Kraft gesetzt, um den dahinter verborgenen inneren Sinn besser zur Geltung kommen zu lassen ${ }^{60}$. Scotus preist (ähnlich wie Thomas in der ,Summa ${ }^{61}$ ) die Einfachheit und Sparsamkeit des Neuen Gesetzes gegenüber dem Alten auf der Ebene der Rechts- und Zeremonialvorschriften. So habe Christus bloß sieben Sakramente eingesetzt, von denen nur zwei (Taufe und gegebenenfalls Buße) heilsnotwendig seien. Allein die explizite Verpflichtung zum Beichtbekenntnis vor dem Priester könnte in ihrem Bereich als Erschwernis angesehen werden. Allerdings zerstreut Scotus die Bedenken mit dem Hinweis auf die (Gnaden-)Hilfen sowie das strenge Beichtgeheimnis ${ }^{62}$. Petrus Aureoli fügt hinzu, daß bereits der sündentilgende Charakter, der dem Bekenntnis an sich innewohnt, dessen belastende Scham ausgleicht ${ }^{63}$. Nochmals ähnlich wie Thomas betont Scotus, daß Christus im Neuen Gesetz keine exakten Festlegungen in Bezug auf Rechtsnormen getroffen und

tertius a distinctione vigesima sexta ad quadragesimam, d. 40, q. un., n. 22, ed. J. Rodríguez Carballo (Opera omnia X), Città del Vaticano 2007, 353sq.: „Amor autem et praecipue finis, si ille in omnibus quaeratur, facit omnia onera levia“.

57 Bonaventura, 3 Sent., d. 40, q. 3 ad 6, ed. Bello (nt. 15), 904a-b.

58 Cf. Thomas, 3 Sent., d. 40, a. 4, qa. 3 sol. und ad 1, ed. Moos (nt. 20), 1311sq. Ein originelles Argument für die Rücknahme der Scheidungserlaubnis durch Jesus ist am Ende des Mittelalters John Mair eingefallen: Wer seiner Frau einen Scheidebrief ausstellt, bekommt vielleicht anschließend eine noch schlimmere (3 Sent., d. 40, q. 4, dub. 2, Paris 1517, 164rb).

59 Cf. Wilhelm von Auxerre, Summa aurea, 1. IV, tr. 3, c. 2 (Spicilegium Bonaventurianum 20), 59, Z. 77-79. Die Hinzufügung einiger Gebote auf „litteraler“ Ebene zählten hier nicht. Cf. auch Summa Halensis, 1. III, n. 560, Quaracchi 1948, 865a-867b.

60 Richard von Mediavilla, 3 Sent., d. 40, a. 2, q. 4 ad 4, Brixen 1591, 487a-b.

61 Cf. Thomas, Summa theologiae, I-II, q. 107, a. 4, Rom 1962, 1044. Im Hintergrund steht bei beiden Augustinus, Ad inquisitiones Ianuarii I, c. 2, n. 2, ed. A. Goldbacher (Corpus Scriptorum Ecclesiasticorum Latinorum 34), Wien 1970, 160.

62 Scotus, Ordinatio, 1. III, d. 40, q. un., n. 12, ed. J. Rodríguez Carballo (nt. 56), 348: „difficile enim videtur alicui peccata privata, soli Deo et sibi nota, alicui homini revelare; sed ibi adbibita sunt tot remedia, quod propter talem revelationem non oportet eum confundi, - obligatur enim audiens ad maximum sigillum secreti."

${ }^{63}$ Cf. Petrus Aureoli, 3 Sent., d. 40, q. ult., a. un., Rom 1605, 541a-b. 
deren weitere Ausgestaltung den Menschen überlassen hat, vor allem den weltlichen wie geistlichen Verantwortungsträgern ${ }^{64}$. Allerdings gibt er anders als dieser zu, daß den Christen damit faktisch sogar eine größere Belastung als zuvor den Juden erwachsen kann, weil sie jetzt nicht mehr bloß den Gesetzen von Priestern und Richtern, sondern zusätzlich den Weisungen weltlicher Fürsten folgen müssen ${ }^{65}$.

(b) Zweitens kann die Tatsache, daß auch das Gesetz des Evangeliums Furcht einflößt, gegen die Überlegenheitsthese ins Spiel gebracht werden. Da sich die Drohungen (analog zu den Verheißungen) nun nicht mehr auf zeitliche Strafen und Verluste, sondern auf die ewige Verdammnis beziehen, sind sie nach Bonaventura sogar objektiv ernster geworden. Und dennoch, so ist er überzeugt, entstand durch das Alte Gesetz real größere Furcht, da zeitliche Strafen den „fleischlichen“ Mensch subjektiv eher erschrecken als ewige: „wie viele Räuber den Diebstahl eher wegen Galgen und Strick fürchten als wegen der Höllenstrafe“ ${ }^{\text {666 }}$. Natürlich ist dabei wiederum die neue Situation in Rechnung zu stellen, die mit Christi Kommen durch die Gewährung der tröstenden Gnadenhilfen und die Möglichkeit echter Vergebung ${ }^{67}$ eingetreten ist.

(c) Ein dritter Problemkomplex eröffnet sich durch die Feststellung, daß manche der alttestamentlichen Gebote in sich bereits jene Vollkommenheit zu besitzen scheinen, die doch angeblich eigentlich erst im Neuen Bund erreicht werden soll, sofern sie nämlich nicht bloß die äußeren Handlungen, sondern auch den menschlichen Willen als ihren innerlichen Grund betreffende Weisungen darstellen. Das von den Scholastikern gerne zitierte Väterwort, wonach das Alte Gesetz nur „die Hand, nicht das Herz" zügle, erweist sich in diesem Zusammenhang als zweifelhaft. Petrus Lombardus hatte diese Problematik mit Blick auf die beiden letzten Gebote des Dekalogs aufgeworfen, wobei er den Dekalogerörterungen bei Hugo von S. Viktor oder in der ,Summa Sententiarum ${ }^{68}$ folgte. Die Antworten der Theologen auf diesen Einwand zielen schon seit der Frühscholastik meistens auf das Zugeständnis ab, daß es tatsächlich bereits im Alten Bund Gesetze gab, die bei rechtem Verständnis Weisungen für das „Herz“ und nicht bloß für die „Hand“ darstellten ${ }^{69}$. Menschen, die sie in dieser geistlichen Weise auszulegen

64 Cf. Thomas, Summa theologiae, I-II, q. 108, a. 2 c. und ad 4, Rom 1962, 1046sq. Die These kennt schon Alexander von Hales, cf. Marcolino, Das Alte Testament (nt. 1), 281.

65 Cf. Scotus, Ordinatio, 1. III, d. 40, q. un., n. 16-17, ed. J. Rodríguez Carballo (nt. 56), 349sq. Das nüchterne Fazit lautet: „Sic ergo, breviter, pauciora sunt onera Legis christianae in quantum sunt tradita a Christo, sed forte plura in quantum post addita sunt alia per eos qui habent regere populum christianum; et ad omnia talia de novo statuta ab Ecclesia tenemur" (n. 17, 350).

66 Bonaventura, 3 Sent., d. 40, q. 1 ad 6, ed. Bello (nt. 15), 898b: „Sicut multi latrones magis timent furari propter patibulum et suspendium quam propter infernale supplicium."

${ }^{67}$ Cf. Summa Halensis, 1. III, n. 552, Quaracchi 1948, 855a-b, ad 1-3.

68 Cf. Hugo von S. Viktor, De sacramentis, 1. I, p. 12, c. 7 (PL 176), 358D-359B; Summa Sententiarum, tr. 4, c. 6 (PL 176), 124D-125A.

69 Dazu Landgraf, Die Gnadenökonomie (nt. 21), bes. 26-30, der auf die Existenz der Formel bei Summisten des 12. Jahrhunderts hinweist. 
wußten, so sagt die ,Summa Sententiarum', gehörten in Wahrheit zum Neuen Bund $^{70}$. Die Überzeugung, daß die beiden Bundesordnungen im Hinblick auf konkrete Einzelpersonen und deren Verständnis der göttlichen Heilsabsicht füreinander durchlässig waren, wird auch von den Autoren des 13. Jahrhunderts durchgängig vertreten ${ }^{71}$. Solche Menschen haben begriffen, daß „das Gesetz mit dem Evangelium schwanger ging ${ }^{672}$, sie haben es aus Liebe befolgt ${ }^{73}$ und wurden dadurch gerettet. Bonaventura geht so weit zu behaupten, daß Gott hauptsächlich ihretwegen das Alte Gesetz erlassen hat ${ }^{74}$. Der großen Masse des Volkes aber fehlte dieser Tiefenblick. Ihr wird im Alten Bund nur eine Gesetzesbefolgung aus Furcht vor Strafe zugeschrieben, die nach Bonaventura Kennzeichen des status imperfectionis ist. Darum fehlte dem Alten Gesetz „in hauptsächlicher Weise“ die Ausrichtung auf die Liebe ${ }^{75}$. Damit kehrt man zu den schon referierten Grundaussagen über die zumeist ausbleibende rechtfertigende Kraft der lex vetus zurück.

(d) Ein vierter Einwand gegen die größere Vollkommenheit des Neuen Bundes beruft sich auf die Abstufung, welche die Kirche ihren Gläubigen hinsichtlich der evangelischen Vollkommenheitsweisungen zugesteht. Demnach sind offensichtlich nicht alle Christen gehalten, das Evangelium in gleicher Weise „ganz“ zu erfüllen wie die Juden ihr Gesetz. Nach Alexander von Hales ${ }^{76}$ ist der Grund für diese Diskrepanz darin zu finden, daß das Evangelium ,,auf Gnade und Liebe hin (in gratiam et amorem)" gegeben sei, nicht aber als Strafe. Deswegen müsse die Erfüllung des vollen Evangeliums stets ein Akt der Freiheit sein. Die Vollkommenheit, zu der die Christen geführt werden sollen, wird in einer Stufenordnung betrachtet, innerhalb derer von einer für alle geltenden Basis aus dem einzelnen ein Aufstieg in freier Entscheidung möglich bleibt. Alexander unterscheidet eine „hinreichende Vollkommenheit" (perfectio sufficientiae), zu der alle berufen sind, von einer Vollkommenheit des Weihestandes (perfectio ordinis), welche für die Geweihten gilt, und einer Vollkommenheit des Ordensstandes (perfectio religionis), welche

70 Cf. Summa Sententiarum, tr. 4, c. 6 (PL 176, 124C-D).

71 Cf. etwa Wilhelm von Auxerre, Summa aurea, l. IV, tr. 2, c. 2 ad 3 (Spic. Bon. 20), 23; tr. 3 , c. 2, 59; Bonaventura, 3 Sent., d. 40, q. 1, ed. Bello (nt. 15), 897a; Thomas, 3 Sent., d. 40, a. 4, qa. 1 und 2, ed. Moos (nt. 20), 1310sq.; Summa theologiae, I-I, q. 107, a. 1 ad 2, Rom 1962, 1041: „Fuerunt tamen aliqui in statu veteris testamenti habentes caritatem et gratiam spiritus sancti, qui principaliter expectabant promissiones spirituales et aeternas. Et secundum hoc pertinebant ad legem novam. Similiter etiam in novo testamento sunt aliqui carnales nondum pertingentes ad perfectionem novae legis, quos oportuit etiam in novo testamento induci ad virtutis opera per timorem poenarum, et per aliqua temporalia promissa“; Richard von Mediavilla, 3 Sent., d. 40, a. 1, q. 3 c., Brixen 1591, 482b; a. 2, q. 4 ad 2, 487a.

72 Bonaventura, 3 Sent., d. 40, q. 2 c., ed. Bello (nt. 15), 900a: ,et qui spiritualiter Legem observabant viri evangelici erant, quia Lex praegnans erat Evangelio."

73 Cf. etwa Thomas, 3 Sent., d. 40, a. 4, qa. 1 ad 1, ed. Moos (nt. 20), 1310, und qa. 2 ad 2, 1311: ,illi qui in veteri lege ex amore legem observabant, perfecti erant; unde ad legem novam pertinebant, in qua est status perfectionis“. Dazu: Guindon, La pédagogie de la crainte (nt. 19), 107-110.

74 Cf. Bonaventura, 3 Sent., d. 40, q. 2 ad 2, ed. Bello (nt. 15), 901b: ,propter spiritualiter intelligentes, qui salvabantur et merebantur in ipsius Legis observatione."

75 Cf. Bonaventura, 3 Sent., d. 40, q. 1 c. und ad 4, ed. Bello (nt. 15), 898a.

76 Cf. Alexander von Hales, Glossa, l. III, d. 40, Quaracchi 1954, 549sq. 
nur diejenigen betrifft, die ein Gelübde abgelegt haben. Nur die letztgenannte Stufe zielt „Vollkommenheit in jeder Hinsicht“ (omnimoda perfectio) an, sofern sie auf die Erfüllung des gesamten Evangeliums gerichtet ist. Alexanders franziskanisches Selbstbewußtsein dokumentiert sich in der hinzugefügten Bemerkung, daß die Nachfolger der Apostel (anders als die Apostel selbst) nicht unter der zuletzt genannten höchsten Verpflichtung stehen, es sei denn, sie gehören zum Orden der Minderbrüder ${ }^{77}$.

\section{Fazit}

Unser Überblick soll mit einigen thesenartigen Feststellungen abschließen.

(1) Der Gesetzesvergleich, den wir im Spiegel einiger Sentenzenkommentare der Hochscholastik skizziert haben, bietet viel Übereinstimmung und wenig kontroverse Inhalte. Die Grundaussagen sind schon in der Frühscholastik vorbereitet und werden von den Theologen des 13. Jahrhunderts nur geringfügig variiert. In der Erläuterung klarer, kaum Spielraum lassender Vätervorgaben hat sich im Mittelalter ein Lehrstück herausgebildet, dem nur wenig echtes theologisches Entwicklungspotential innewohnte.

(2) Deutlich erkennbar ist der Wille der Autoren, trotz aller negativen Urteile über die Effekte des Alten Gesetzes die göttliche Urheberschaft hinter beiden Testamenten gegen jede Marcionismustenden ${ }^{78}$ zu verteidigen. Dies zeigt sich nicht nur in den ausführlichen und wertschätzenden Erörterungen, die Theologen des 13. Jahrhunderts dem Alten Gesetz bzw. besonders wichtigen Einzelvorschriften und Sakramenten (wie etwa der Beschneidung) nach ihrer Bedeutung in derjenigen Epoche der Heilsgeschichte gewidmet haben, für die sie nach dem Willen Gottes Geltung besitzen sollten. Auch im Vergleich mit dem Neuen Bund kann durch das beide Gesetze verbindende Schema von Verheißung und Erfüllung eine enge Verbindung der Ordnungen problemlos zugestanden werden. Letztlich, so Thomas in der ,Summa', ordnen Altes und Neues Gesetz den Menschen auf das gleiche Ziel hin, die Unterordnung unter Gott, und darum sind sie der Art nach identisch, erfüllen ihre Aufgabe allerdings in unterschiedlicher (unvollkommener beziehungsweise vollkommener) Form ${ }^{79}$. In diesem Zusam-

77 Cf. ibid., 550.

78 Cf. sehr deutlich Matthaeus ab Aquasparta, Quaestiones de legibus, q. 4, ed. Piana (nt. 31), 494-518.

79 Thomas, Summa theologiae, I-II, q. 107, a. 1. Ähnlich bereits Summa Halensis, 1. III, n. 547, Quaracchi 1948, 845a-846b: „Dicendum quod lex Evangelii et lex Moysi una lex est $<$ in $>$ ratione universali, diversae vero sunt in ratione propria. Unde Augustinus, Contra adversarium Legis et Prophetarum 4: ,Qui recte colit Deum, utriusque Testamenti invenit unum Deum, et eiusdem unius Dei in utroque diligit bonitatem, in utroque metuit severitatem" (845b). Cf. nach Thomas Richard von Mediavilla, 3 Sent., d. 40, a. 2, q. 4 c., Brixen 1591, 486b: „Finis enim utriusque legis est, ut homines sic subdantur Deo ut iustificentur supernaturali iustitia, qua digni sunt praemio transcendente $[\mathrm{m}]$ nostram naturalem facultatem, quod est vita aeterna“; Petrus Aureoli, 3 Sent., d. 40, q. ult., a. un., Rom 1605, 541a. 
menhang werden durch die scholastischen Autoren einerseits zahlreiche Klischees hinsichtlich der Inferiorität jüdischer Religiosität aus der patristischen Literatur recht unkritisch tradiert, andererseits wird jedoch das Motiv der Einheit des göttlichen Heilsplans und der Kontinuität des einen Gottesvolkes, der ecclesia ab Abel, deutlich bestätigt ${ }^{80}$.

(3) Wenn die Theologen dem Alten Gesetz einen Eigenwert zugestehen, dann stets nur bis zum Tod Christi, dem alles Vorangehende überbietenden eigentlichen Erlösungsereignis. Vergeblich sucht man im Zusammenhang unserer Fragestellung nach positiven Einschätzungen des alttestamentlichen Gesetzes und des ihm treu bleibenden Judentums für die Zeit nach Christus. Als schlechterdings „tötendes Gesetz“ wird es nun auf jeden Fall hinsichtlich seiner Kultvorschriften charakterisiert, deren Befolgung in der Zeit des Evangeliums als Häresie und Todsünde qualifiziert werden $\mathrm{kann}^{81}$. Hinsichtlich der Rechtsnormen war eine Diskussion darüber möglich, ob sie im Neuen Bund weiterhin nützlich (,Summa Halensis?) oder zumindest als indifferent, weder wirksam noch schädlich, anzusehen sind, da ihnen schon immer echte religiöse Relevanz gefehlt hat (so die Meinung des Durandus) ${ }^{82}$. Daß den Moralnormen (vor allem dem Dekalog) dauerhafte Geltung zugestanden wird, hängt weniger mit ihrer Promulgation im Alten Bund als mit dem ihnen zugeschriebenen naturrechtlichen Charakter zusammen. Indem der Begriff des Gesetzes im Neuen Bund somit unter den Bedingungen einer radikalen materialen Beschränkung zu betrachten ist und durch die enge Verbindung mit dem Konzept der sich nun darin ausdrückenden inneren Gnade vor allem unter einer veränderten formalen Perspektive erfaßt wird, läßt sich die Frage stellen, ob er im Gegensatzpaar lex vetus vs. lex nova tatsächlich noch uneingeschränkt univok verwendet wird. Insgesamt sehen sich die scholastischen Autoren von diesen Prämissen her nur in der Lage, die Weiterbefolgung des Gesetzes durch die Juden als Inbegriff heilsgeschichtlichen Unverstandes und atavistischer Religionsausübung anzusehen.

(4) Aus der Perspektive der mittelalterlichen Diskussion unseres Themas muß es eigentlich erstaunen, daß die Gegenüberstellung von Gesetz und Evangelium in der Reformationszeit zu einem Topos innerchristlicher Polemik werden konnte, der bis weit in die Moderne hinein die Kontroverstheologie geprägt hat. In ihr ist gerade die mittelalterlich geprägte katholische Tradition häufig mit dem Vorwurf einer Vergesetzlichung des Evangeliums konfrontiert worden. In den von uns untersuchten scholastischen Texten ist als Ansatzpunkt dafür bestenfalls das von Augustinus her geläufige Zugeständnis auszumachen, daß nicht nur im Alten

80 Cf. auch Marcolino, Das Alte Testament (nt. 1), 137-174.

81 Cf. Summa Halensis, 1. III, n. 542, Quaracchi 1948, 832-836; Matthaeus ab Aquasparta, Quaestiones de legibus, q. 6 c., ed. Piana (nt. 31), 550.

82 Durandus, 3 Sent., d. 40, q. 3, Paris 1550, 247vb. Cf. mit ähnlicher Richtung bereits Wilhelm von Auxerre, Summa aurea, 1. III, tom. 2, tr. 44, c. 4 ad 2, ed. J. Ribaillier (Spicilegium Bonaventurianum 18 B), Paris-Grottaferrata 1986, 843, Z. 46-47. 
Bund manche Menschen durch ihr Gesetzesverständnis faktisch zum Neuen gehörten, sondern auch Umgekehrtes gelten kann ${ }^{83}$. Unterscheidungen zwischen den Christen nehmen die Theologen des Mittelalters jedoch nicht von diesem Maßstab aus vor, sondern viel eher mit Blick auf die mehr oder weniger intensiv ausgeprägte Bereitschaft des Einzelnen, sich der Vollkommenheitsforderung des Evangeliums zu stellen. Daß die Vollkommenheit im Neuen Bund keine Frucht gesetzlich konzipierter Frömmigkeit ist, sondern aus dem Geschenk der Gnade und Liebe Christi hervorgeht und in der Antwort eines Lebens aus Glaube und Liebe sichtbar wird, steht in der Hochscholastik außer Zweifel.

83 Cf. die in nt. 71 zitierten Texte. 